\title{
Detection of Plant Diseases Using Image Processing Tools -A Overview
}

\author{
Asha R. Patil Varsha I.Patil , B.S.Panchbhai \\ S.T.Co.Op.College Shahada, PSGVPS College Shahada, R.C.Patel College, Shirpur
}

\begin{abstract}
Analysis of plants disease is main goal for increase productivity of grain, fruits, vegetable.

Detection of proper disease of plants using image processing is possible by different steps of it. Like image Acquisition, image enhancement, segmentation, feature extraction, and classification.RGB image is acquire and translate for processing and diagnosis of plant disease by CR-Network. Segmentation is used for which and how many areas are affected by disease using k-clustering. Future extraction by HOG algorithm, SOFM Classification is used for healthy and unhealthy plants
\end{abstract}

Keywords: Image Acquisition, RGB image, CR-Network, HOG algorithm, SOFM Classification

\section{INTRODUCTION}

India is fast growing population country and ratio of food grain production is as compare to low. India has over 210 million acres of farm land [7]. In India many people working in the agriculture sector. The agricultural sector plays crucial role in rural area development. It means need of research in agriculture field is very important now a day in fast speed. Indian researcher is fastly growing in this field. Plant diseases situation is dilemma for farmer. Plant disease is harmful for plant and production of food grain. Farmers identify disease symptoms by naked eyes, it is very difficult and need continuous observing of that plants. It very difficult practically for every farmer. In this paper there are some researchers who define problem as well as their solution in different way. If detection of Plant disease is earlier then treatment is also fastly taken or may take prevention better. Detection of plant disease is challenging work for researcher. Causes are different namely envoirment, global warming, pollution, number of viruses and bacteria, fungi's and so many. Now a day farmer need consult about crop diseases, advance pesticide and environmental changes with agriculture experts. Plant has different types of diseases related to leaf, step, fruit. Identify proper disease and its causes is important research for researcher

\section{LITERATURE SURVEY}

Jagadees[1] proposed system focused on early detection and classification of fungal disease on different agriculture plants fruit crop, vegetable crop and some commercial crop by using techniques of image processing segmentation, feature extraction ,classification .Fungal diseases symptom of plant namely anthracnose, powdery mildew, downey mildew affected on mango, pomegranate ,grape are categorized into partially, moderately ,severally affected.

Jundare Manisha [2] her proposed system based on two aspect client side and server side. To identification of infected area of plant leaves image is capture by camera and send server side using CRNetwork algorithm HOG algorithm. After uploading image server identify, classify disease of plant and suggest appropriate treatment provided using SVM (Support Vector Machine) and arrangement Algorithm, Otsu Threshold Algorithm. This research method is fastest and effective in detection of diseases of plants.

Suvarna [3] in her research method image sampling is done by image acquisition and after getting images noise are removed and enhance the image. Enhance Image is convert into binary image and extract its color features, hence affected area is estimated. Then classification and grading is done.

The final result consists of numerical values of total number of red pixels, total number of pixels covered by the chilli fruit, Total area affected by the disease.

Smita Naikwadi [4]The author of this paper finding the disease of plant by various image processing methods namely image acquisition, image transformation, clustering, thresholding feature extraction, texture analysis and histogram. Sample RGB image is transform into HIS color image. Using different algorithm and matlab function calculate the histogram of images. Histogram is generating sample image and testing image. Both images are comparing using classification. Using GUI see the process of result. K. Jagan Mohan [5] his proposed system works on paddy plants diseases detection. He also uses various image processing techniques like image capturing by camera or mobile, image pre-processing for affected area is manually cropped. Feature extraction is done 
by HOG algorithm and for classification it used SVM machine .Overall work is applicable for paddy plant diseases namely Brown spot, Leaf blast and Bacterial blight. Sujeet Varshney [6] in his proposed work he used Support Vector machine SVM algorithm.RGB image is capture and infected part is identify dataset values. self-organising feature map (SOFM) of neural network and pattern classification are detecting the diseases of plants.

\section{CONCLUSION}

In above discussion gather all information about diseases of plants so I conclude that image processing is one of the important tools for disease detection of plants. Some algorithms are give efficient result for treatment of pesticide and preserve from major attack of viruses, bacteria, and others. All proposed method help to next researcher to advance finding of diseases for prevention.

\section{REFERENCES}

[1]. Jagadeesh. D. Pujari Reduced Color and Texture features based Identification and Classification of Affected and Normal fruits' images International Journal of Agricultural and Food Science -2013.

[2]. Jundare Manisha. A. Jundare Pallavi T. Jundare Pragati V. Prof. C.S.Aryan, Plant Disease Detection and its Treatment using Image Processing ,International Journal of Advanced Research in Electrical, Electronics and Instrumentation Engineering- 2016.

[3]. SUVARNA KANAKARADDI ANALYSIS AND GRADING OF PATHOGENIC DISEASE OF CHILLI FRUIT USING IMAGE PROCESSING International Conference on Advances in Engineering \& Technology- 2014.

[4]. Smita Naikwadi, Niket Amoda, ADVANCES IN IMAGE PROCESSING FOR DETECTION OF PLANT DISEASES, International Journal of Application or Innovation in Engineering \& Management 2013.

[5]. K. Jagan Mohan, Recognition of Paddy Plant Diseases Based on Histogram Oriented Gradient Features International Journal of Advanced Research in Computer and Communication Engineering -2016.

[6]. Sujeet Varshney, A Novel Approach for the Detection of Plant Diseases, International Journal of Computer Science and Mobile Computing -2016.

[7]. Vyshnavi.G.K, Sirpa.M. Chandramoorthy, Padmapriya. Healthy and Unhealthy Plant Leaf Identification and Classification Using Hierarchical Clustering, International
Research Journal of Engineering and Technology 